\title{
Caffeine Toxicity Following Ingestion of an Exercise Supplement by a Patient with Type 1 Diabetes
}

\author{
Ehab Hamed \\ Family Medicine Consultant, Primary Health Care Corporation, Doha, Qatar
}

How to cite this article: Hamed E. Caffeine toxicity following ingestion of an exercise supplement by a patient with type 1 diabetes. EJCRIM 2018;5: doi:10.12890/2018_000957.

Conflicts of Interests: The Authors declare that there are no competing interests.

This article is licensed under a Commons Attribution Non-Commercial 4.0 License

\section{ABSTRACT}

We report the case of a patient with type 1 diabetes who developed acute severe diabetic ketoacidosis following ingestion of an energy supplement containing caffeine. Some $95 \%$ of the US adult population consume caffeine, and the general perception is that there are no negative consequences for health. The upper limit of safe consumption is less than $400 \mathrm{mg}$ per day. However, acute ingestion of high doses of caffeine may cause significant metabolic changes that can be fatal. Here the patient consumed a toxic dose of caffeine causing unpleasant and puzzling symptoms, vomiting and, following omission of his long-acting basal insulin, severe diabetic ketoacidosis. As the sports nutrition market continues to expand, providers and manufacturers have a responsibility to give clear and accurate dosing instructions as well as side effect profiles for their products, particularly for diabetic patients.

\section{LEARNING POINTS}

- The impact of caffeine on blood glucose levels has implications for people with diabetes who may be thinking of consuming supplements containing caffeine.

- Providers of sports supplements have a responsibility to provide an accurate description of the side effects with a clear warning for diabetic patients

- Legislators should review protocols for regulating the sports and nutritional supplements industry.

\section{KEYWORDS}

Caffeine toxicity, diabetes, diabetic ketoacidosis

\section{CASE DESCRIPTION}

The patient was a 28-year-old male with a 16-year history of type 1 diabetes treated with multiple short acting daily injections and background basal insulin at night and rapid-acting analogue insulin with each meal. Overall diabetes control based on HbA1c levels was suboptimal (range 8-12\%). The patient decided that regular exercise might be beneficial and so joined a local gym. However, before taking up exercise, he purchased a training supplement from an internet-based supplier advertised as 'pure caffeine powder' which had the potential to increase metabolism and improve fat oxidation. The background label says 'boost your workout, increase metabolism'. Online instructions provide a dosage regimen which recommends $200 \mathrm{mg}$ of caffeine powder one to three times daily. A measuring scoop is provided at an additional price. While these directions are available on the product website, the packaging itself does not mention the recommended dose. Consumers are advised to seek medical attention if adverse events occur. 
Online customer reviews reflect the potential for harm, including admission to hospital. One consumer mixed an excessive amount of pure caffeine powder with another supplement containing protein. He reports becoming rapidly unwell, resulting in hospital admission. He warns others of the potential effects but still indicates that he is pleased with the product when taken at the correct dosage.

Our patient ingested $5 \mathrm{~g}$ (equivalent to 25 cups of coffee) of the product before exercise, following which he became acutely agitated and was too unwell to exercise. Subsequently, he experienced palpitations, abdominal cramps, chest tightness and vomiting. Although he continued with his regular doses of rapid-acting insulin, because of perceived concerns about nocturnal hypoglycaemia, he omitted the evening dose of long-acting basal insulin.

The following morning, the patient was admitted to hospital with diabetic ketoacidosis (arterial blood glucose $39 \mathrm{mmol} / \mathrm{l}, \mathrm{pH} 7.2$, base excess -19.5), but made an uneventful recovery after 2 days receiving a standard protocol. The treatment regime included intravenous fluids and insulin, potassium supplementation and regular measurements of acid-base status and blood ketone levels.

\section{DISCUSSION}

Numerous performance-enhancing supplements are available on the internet and as over-the-counter products, providing the public with easy access to high doses and bulk quantities. Caffeine is a component of many of these products as it can enhance performance ${ }^{[1]}$. The literature and internet reviews suggest that public and fitness fans use these supplements in excessive and potentially dangerous doses, resulting in many hospital admissions and at least 51 fatalities ${ }^{[2]}$. The literature states that a fatal dose is about $5-10 \mathrm{~g}$ or $150-200 \mathrm{mg} / \mathrm{kg}$ body weight $^{[3]}$. In diabetic patients, acute ingestion of excessive amounts of caffeine or sports drinks may be associated with hyperglycaemia ${ }^{[3,4]}$. Unintentional and intentional harm due to caffeine overdoses is becoming more frequent .

Here the patient consumed a harmful dose of caffeine causing unpleasant, noxious symptoms and vomiting, either through a direct effect or indirectly because of raised blood sugar. The patient then compounded the problem by omitting his long-acting basal insulin, which resulted in acute hospital admission with severe diabetic ketoacidosis that required 2 days for recovery. In our view, the toxic dose of caffeine caused the problem, which was then further complicated by omission of the insulin.

The potential impact of caffeine on blood glucose levels has implications for patients with diabetes who may be thinking of consuming products containing caffeine and marketed as an 'energy supplement'. Our patient's use of this caffeine supplement and his management of the subsequent hyperglycaemia was unwise. Consumers must accept responsibility when taking these supplements and ensure they are following product guidelines.

We also believe that providers have a responsibility to provide a clear warning about the adverse consequences of excessive dosing for consumers and in particular, for diabetic patients. They should refrain from poor labelling and misleading marketing practices. Also, regulators should ensure appropriate labelling is used and should consider limiting public access to bulk purchases. Ease of access to highdosage pure caffeine poses a public threat to diabetic as well as healthy individuals.

\section{REFERENCES}

1. USDA. Dietary Guidelines for Americans 2015-2020. 8th ed. U.S. Department of Health and Human Services and U.S. Department of Agriculture; 2015. 144 p. Available from: http://health.gov/dietaryguidelines/2015/guidelines/ (accessed 1 October 2018).

2. Jones AW. Review of caffeine-related fatalities along with postmortem blood concentrations in 51 poisoning deaths. J Anal Toxicol 2017;41:167-172.

3. Kerr Everett DJ. Coffee, diabetes and insulin sensitivity. Diabetologia 2005;48:1418.

4. Olateju T, Begley J, Green DJ, Kerr D. Physiological and glycemic responses following acute ingestion of a popular functional drink in patients with type 1 diabetes. Can J Diabetes 2015;39:78-82. 